\title{
Determination of optimum operation cases in electric arc welding machine using neural network ${ }^{\dagger}$
}

\author{
Tahir Çetin Akinci ${ }^{1, *}$, Hidir Selçuk Noğay ${ }^{2}$ and Gökhan Gökmen ${ }^{3}$ \\ ${ }^{1}$ Faculty of Technology, Department of Electrical and Electronics Engineering, Kirklareli University, Kirklareli, Turkey \\ ${ }^{2}$ Faculty of Technical Education, Department of Electrical Education, Kirklareli University, Kirklareli, Turkey \\ ${ }^{3}$ Faculty of Technical Education, Department of Electrical Education, Marmara University, Istanbul, Turkey
}

(Manuscript Received August 21, 2010; Revised November 26, 2010; Accepted January 2, 2011)

\begin{abstract}
With arc welding machines, welding is only performed at optimum operating points. Determination of optimum operating points is important so as for welding machines which will be produced in future to be developed in a manner to operate in such parts. In this study, an Artificial Neutral Networks method was used in order to determine the optimum operating points of Electric Arc welding machine. For this purpose, a measurement system used to get the current measurements during the welding operation. A welding process includes some stages like initial case; transient case and operation case respectively. So as to use ANN model, a data set was established via time series. ANN is trained with $90 \%$ of data set and tested with $10 \%$ thereof. At the end of the test, a prediction of $97.49 \%$ was made according to the regression value. And according to the MSE value, it was understood that a successful prediction was made with an error of 0.00353075 values.
\end{abstract}

Keywords: Electrical arc welding machine; Optimum operation cases; Time series; Welding analysis

\section{Introduction}

The main elements of the welding are temperature and pressure. Welding has its roots in Bronze Age. In those periods, for the fire and welding methods were used to be utilized for the production of weapon and similar tools. In today, welding is performed in the ship, bridge, building construction and automotive sector using modern methods [1-3]. Welding varies depending on the material type to be welded and application field. For this study, MAG welding method was used. MAG welding is welding in an atmosphere of reacting gases, or as it is also called, under cover of an active gas. This means that the gas splits in the electric arc and reacts with the weld pool to a lesser or greater extent. Advantages of MAG welding;

- MAG welding method is very productive and economical welding.

- This method is very useful in industry.

- MAG procedure is used for welding stainless steel, aluminum, cooper or cooper alloys and also magnesium, nickel and a number of other metals and their alloys.

\footnotetext{
${ }^{\dagger}$ This paper was recommended for publication in revised form by Associate Editor Yong-Tae Kim

${ }^{*}$ Corresponding author. Tel.: +90 288 2461666, Fax.: +90 2882461650

E-mail address: cetinakinci@hotmail.com

(C) KSME \& Springer 2011
}

- The method provides the opportunity for rational welding of materials which are difficult to weld.

- In this method welding is possible in all positions.

Welding equipment for MAG welding consists in principle of; a shielding gas system with control, a power source, a wire feed unit, a complete welding torch and a reef of welding wire.

In order to obtain a stable arc the power source used for MAG welding must have a properly set or adjustable characteristic and an outlet for the appropriate inductance values. Static characteristic is the curve for voltage $(\mathrm{V})$ versus current (A). A normal power source has a falling static characteristic, while a power source with an approximate flat characteristic is usually used for MAG welding. Electrical equipments are the heart of the welding machines [4, 5].

An electric arc formed by a welding power source can be considered as a transformation of electrical energy to heat energy which is required during the ac welding. The arc welding involves low-voltage, high current arcs between the electrode and material as a work piece [6]. Some important applications of the electric arcs in which it is desirable to know the arc parameters accurately are in arc welding, plasma torches, switchgear, and arc furnaces $[7,8]$.

All electric welding machines perform the same function regardless of their size; they melt pieces of metal in order to mechanically bond them together [9-11] 
They output a low voltage alternating current called AC at a high amperage. The voltage range is between 24 and 50 volts $\mathrm{AC}$ while the current value changes from 20 amperes to as high as 500 amperes. Effect of this high current appears by blue arc in welding. This arc at high temperature heats up the metal and then it melts a puddle of molten metal.

The most common welder used in industrial is the arc welder. This type of electric machine uses a stick electrode to conduct the electricity to sample material and hence it melts at the same time to fill in the gaps. A wire feed machine uses a roll of wire and the wire feeds into the blue arc and fills in the gap between the two pieces of metal. A Tungsten Inert Gas (TIG) machine or welder uses a tungsten tip which creates the high temperature needed in the welding process. Along with the arc, an inert gas such as argon is fed into the TIG welding puddle of metal to remove any impurities that come from the surrounding environment [11].

With arc welding machines, welding is only performed at optimum operating points. Determination of optimum operating points is important so as for welding machines which will be produced in future to be developed in a manner to work in such parts. Determination of optimum operation cases can be possible using some methods. There are a lot of methods for forecasting problems. The most effective method is artificial neural network for estimation. Optimum operation points are important for metallurgy science. For this purpose determination of these points of welding machines is simpler using by ANN. So developing ANN model is necessary for future studies.

In this study, an empirical data acquiring system was initially formed and the current drawn by welding machine in 5seconds was measured. Later, current date acquired from data acquiring system was computerized and current drawn by the system - time graphic analysis was made. Within the light of the findings obtained from this analysis, a data set was formed so as to use in artificial neutral networks by means of time series method. This data set was utilized to determine different operating parts of welding machine, a multilayered artificial neutral network. Back propagation learning algorithm was used for the purpose of training. Via methods used, start-up moment, transient parts, operating parts and optimum operating parts of welding machine were predicted.

\section{Artificial neural network (ANN)}

Capability of ANN to learn from experiences and make generalizations are some of the most important features of ANN attracted researches. Because, whether the relation between inputs and outputs regarding an event is linear or not, capabilities of learning from existing samples in hand, making generalization, creating solutions for the unprecedented events constitute the base of smart behavior in ANNs. Also, ANNs have characteristics such as creating solutions within the shortest time making parallel operations since they are actualized as hardware, accessing the original information out of

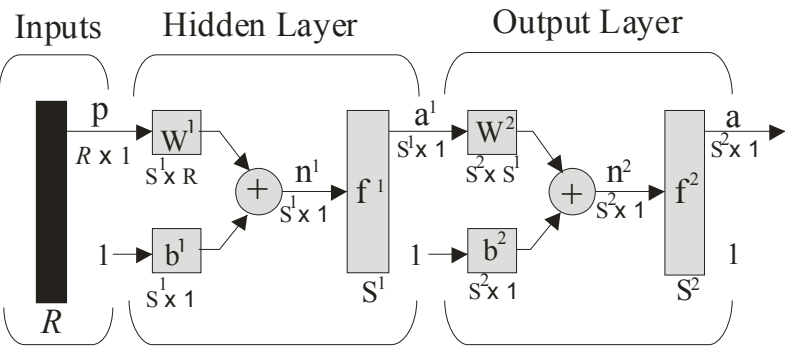

Fig. 1. Two-layers feed forward network.

noisy data, being adopted in a rapid and easy manner in terms of parameter and function, facilitating the solutions of nonlinear complex problems.

There are multitudes of different types of ANN models. Some of the more popular of them include the multilayer perceptron, which is generally trained with the back propagation algorithm. In Fig. 1 two layers feed forward network for general application of ANN is illustrated. Back propagation is a training method for multilayer feed forward networks. Such a network proposed ANN model for this study, including three layers of perceptrons is shown in Fig. 2. In this study thirtyfive number of neuron was used in the hidden layer and sixteen input parameters and five output parameters were used in input layer as shown in Table 1 and Table 2 respectively [12].

By the algorithmic approach known as LevenbergMarquardt back propagation algorithm, the error is decreased repeatedly. Some ANN models employ supervisory training while others are referred to as none-supervisory or selforganizing training. However, the vast majority of ANN models use supervisory training. The training phase may consume a lot of time. In the supervisory training, the actual output of ANN is compared with the desired output. The training set consists of presenting input and output data to the network. The network adjusts the weighting coefficients, which usually begin with random set, so that the next iteration will produce a closer match between the desired and the actual output. The training method tries to minimize the current errors for all processing elements. This global error reduction is created over time by continuously modifying the weighting coefficients until the ANN reaches the user defined performance level. This level signifies that the network has achieved the desired statistical accuracy for a given sequence of inputs. When no further training is necessary, the weighting coefficients are frozen for the application. After a supervisory network performs well on the training data, then it is important to see what it can do with data it has not seen before. If a system does not give reasonable outputs for this test set, the training period is not over. Indeed, this testing is critical to insure that the network has not simply memorized a given set of data, but has learned the general patterns involved within an application $[12,13]$.

The back-propagation learning algorithm is presented below in brief. For each neuron in the input layer, the neuron outputs are given by 


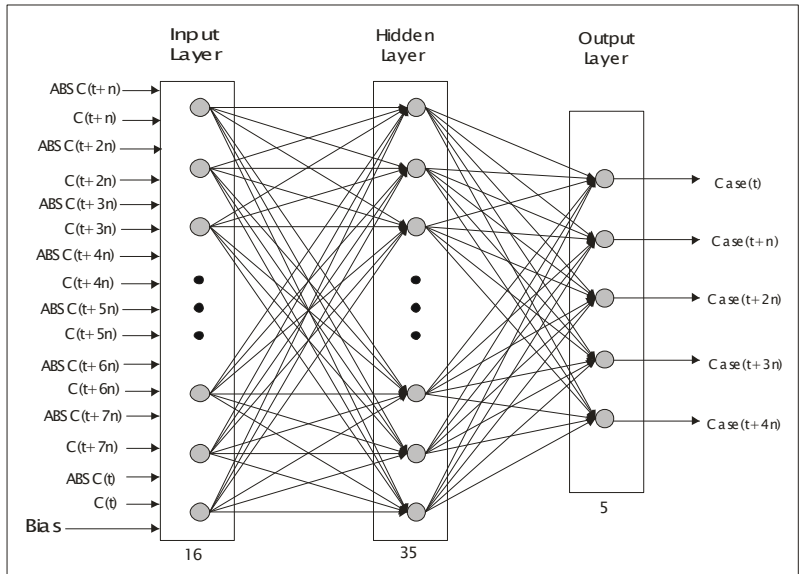

Fig. 2. Proposed ANN Model.

$$
n_{i}=o_{i}
$$

where ni is the input of neuron $i$, and oi the output of neuron $i$. Again for each neuron in the output layer, the neuron inputs are given by

$$
n_{k}=\sum_{j=1}^{N_{j}} w_{k j} o_{j}
$$

where $w k j$, is the connection weight between neuron $j$ and neuron $k$, and $N j, N k$ the number of neurons in the hidden layer and output layer, respectively. The neuron outputs are given by

$$
o_{k}=\frac{1}{1+\exp \left[-\left(n_{k}+\theta_{k}\right)\right]}=f_{k}\left(n_{k}, \theta_{k}\right)
$$

where $\theta k$ is the threshold of neuron $\mathrm{k}$, and the activation function $\mathrm{fk}$ is a sigmoidal function. For the neurons in the hidden layer, the inputs and the outputs are given by relationships similar to those given in Eqs. (2) and (3), respectively.

The connection weights of the feed-forward network are derived from the input-output patterns in the training set by the application of generalized delta rule. The algorithm is based on minimization of the error function on each pattern $p$ by the use of steepest descent method. The sum of squared errors Ep which is the error function for each pattern is given by

$$
E_{P}=\frac{1}{2} \sum_{k=1}^{N_{k}}\left(t_{p k}-o_{p k}\right)^{2}
$$

where $t_{p k}$ is the target output for output neuron $\mathrm{k}$, and $o_{p k}$ the calculated output for output neuron $k$. The overall measure of the error for all the input-output patterns is given by

$$
E=\sum_{p=1}^{N_{p}} E_{p}
$$

where $N_{p}$ is the number of input-output patterns in the training set. When an input pattern $\mathrm{p}$ with the target output vector $t p$ is presented, the connection weights are updated by using the following equations:

$$
\begin{aligned}
& \Delta w_{k j}=\eta \delta_{p k} o_{p j}+\alpha \Delta w_{k j}(p-1) \\
& \delta_{p k}=\left(t_{p k}-o_{p k}\right) o_{p k}\left(1-o_{p k}\right)
\end{aligned}
$$

where $\eta$ is the learning rate, and $\alpha$ is the momentum constant. Again, the connection weights between input layer neuron $i$ and hidden layer neuron $j$ can be updated by using the following equations:

$$
\begin{aligned}
& \Delta w_{j i}=\eta \delta_{p k} o_{p j}+\alpha \Delta w_{j i}(p-1) \\
& \delta_{p j}=o_{p j}\left(1-o_{p j}\right) \sum_{k=1}^{N_{k}} \delta_{p k} w_{k j} .
\end{aligned}
$$

It is important to note that the threshold $\theta$ of each neuron is learned in the way same as that for the other weights. The threshold of a neuron is regarded as a modifiable connection weight between that neuron and a fictitious neuron in the previous layer which always has an output value of unity [14$16]$.

In order to use the ANN simulator for any application, first the number of neurons in the layers, type of activation function (purelin, tansig, logsig), the number of patterns, and the training rate must be chosen.

ANN designing process involves five steps. These are gathering input data, normalizing the data, selecting the ANN architecture, training the network, and validation-testing the network.

\subsection{Time series analysis}

Sequenced data sets acquired from the measures performed at specific time intervals of one or more variables are called as "time series". Prediction can be made regarding future periods using data of a time interval by means of time series.

Time series fluctuate under the effect of many factors affecting it. Such fluctuations occur due to the fact that different factors such as economical, social, demographical, physiological etc. affect time series in different directions and strengths. The most important feature of time series is that changes on observation values occur as a result of common effect of such factors. Such factors can be ranged as Trend (T) Seasonal fluctuation (S), Cyclical fluctuation (C), Irregular (random) fluctuations (I). A time series with such factors can be generally shown with Eq. (10).

$$
\mathrm{Y}=\mathrm{T}+\mathrm{S}+\mathrm{C}+\mathrm{I}
$$

The selection of method to be used in the prediction of future value of time series varies depending on the purpose of the prediction, type and factors, previous data amount of time 


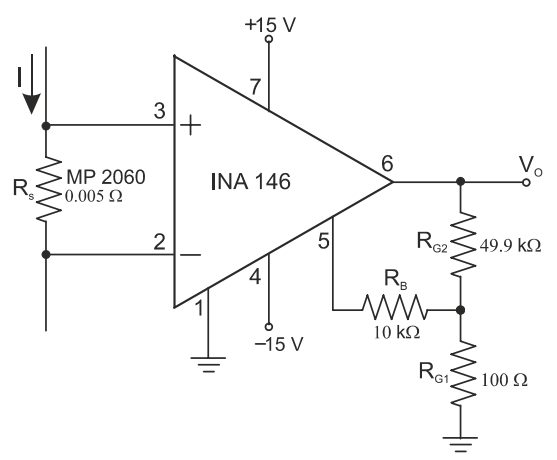

Fig. 3. Current shunt measurement.

series, and length of prediction period. Today, it is feasible to determine the most appropriate model via developed computer softwares. For this study, empirical application time as time series was used. This process is 5 seconds and sampling time is 0.005 seconds. . This sampling time constitutes the lowest structural unit of the times series and was evaluated as a step demonstrated in the Table 1 and Table 2 in order to acquire input and output variations of ANN [13, 17].

\section{Measurement system and data acquisition}

In this study, the measurement of the instantaneous primer current drawn by the welter is realized by the help of a current shunt measurement. In this method, the current which is aimed to measure passes into a resistor and a voltage occurred according to Ohm law. Voltage drop across of the shunt resistor is proportional to measurement current. Shunt voltage amplified by a gain amplifier such as difference amplifier because of low shunt voltage. Sense resistor should be placed between load and source [19, 20]. In this study MP2060 $(0.005 \Omega)$ power film resistor is used as shunt resistor [21, 22] and INA 146 difference amplifier of Burr-Brown Corporation is preferred as gain amplifier Output voltage of the amplifier is proportional to shunt voltage and measurement current. Connection scheme of the current shunt measurement method is given in Fig. 3. [22, 23].

Voltage of the shunt resistor generated by the welding current can be calculated as:

$$
V_{s}=I \cdot R_{s}
$$

In this equation, Vs represents shunt voltage in $\mathrm{V}, I$ represent welding current in A, Rs represents Shunt resistor value in $\Omega$.

Calculation of output voltage of the INA 146 amplifier is given below $[5,6]$ :

$$
V_{o}=V_{s} \cdot\left[0.1\left(1+R_{G 2} / R_{G 1}\right)\right]
$$

In this equation, Vo represent output voltage of the amplifier in V, RG1 and RG2 represent gain adjustment resistor in $\Omega$.

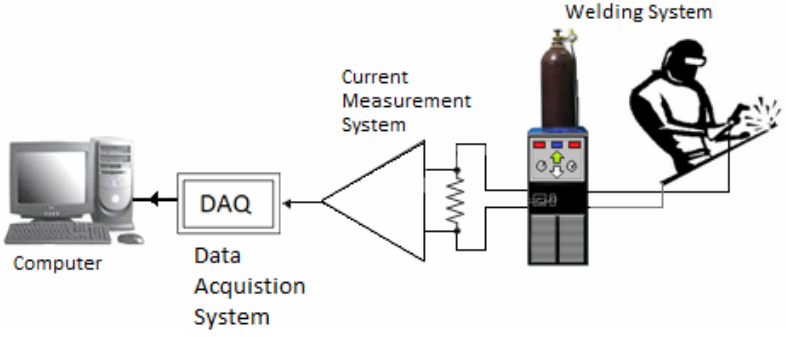

Fig. 4. Measurement and Data Acquisition system for welding.

If gain is adjusted as $50, \mathrm{RG} 1$ is selected as $100 \Omega$ and, RG2 is selected as $49.9 \mathrm{k} \Omega$ [23].

According to this constant value, relationship between welding current and output voltage is:

$$
I=\frac{V_{o}}{R_{s}\left[0.1\left(1+R_{G 2} / R_{G 1}\right)\right]}=4 \cdot V_{o} .
$$

If output voltage is selected $\pm 14.475 \mathrm{~V}$ ( it is almost maximum amplifier output voltage), Maximum welding current value is calculated as $\pm 57.9 \mathrm{~A}$. This value is accepted as $\pm 1 \mathrm{pu}$ for convenience and further measured current values are specified according to that value.

The current shunt measurement's output is sent to the computer system through the PCI hardware, which is multifunction type of Advantech 1716L, with sampling rate at 0.005 sec., and then the collected data is analyzed by MATLAB program. For this purpose, considered measurement and data collection system is shown by Fig. 4 .

Related data used in this study is collected during the welding process of two work pieces as iron material by an electrical arc welding machine (its model is ESAB-LHE 260). In this study Metal Active Gas (MAG) welding method is applied. In addition welding process is made on the ST 37 type material using cored wire with "rutile" basis. As technical information, some specific properties of this welter can be listed as below:

Frequency: $50 / 60 \mathrm{~Hz}$

Number of phases: $3 \sim$ AC.

Primer: 100 VA, Voltage: $440 \mathrm{~V}$ - 220V - 240V

Primer Current: 17/29 A

Seconder: 55V (Dc), \%100:200 A - \%35: 315 A

\section{Gathering the input and output data}

Fig. 5 provides current graphic of the welding machine in the 5-second operating condition that is used for the study. While forming data set, it was based on this graphic. Different operating regimes are seen on this graphic. As seen in the Table 2, a symbolic number corresponding each of different operating conditions was used for the output of artificial neutral network. This symbolic number used is the highest current for each of the operating condition demonstrated in the graphic. Current was used as an input for the places corre- 
Table 1. Input summary of data set.

\begin{tabular}{|c|c|c|c|c|}
\hline Inputs & Max & Min & Points & Number \\
\hline $\operatorname{ABS} C(\mathrm{t}+\mathrm{n})$ & 0,899996 & 0,00088 & $2-982$ & 1 \\
\hline $\mathrm{C}(\mathrm{t}+\mathrm{n})$ & 0,852933 & $-0,89999$ & $2-982$ & 2 \\
\hline $\operatorname{ABSC}(\mathrm{t}+2 \mathrm{n})$ & 0,899996 & 0,00088 & $3-982$ & 3 \\
\hline$C(t+2 n)$ & 0,852933 & $-0,89999$ & $3-982$ & 4 \\
\hline $\operatorname{ABSC}(\mathrm{t}+3 n)$ & 0,899996 & 0,00088 & 4-982 & 5 \\
\hline$C(t+3 n)$ & 0,852933 & $-0,89999$ & $4-982$ & 6 \\
\hline $\operatorname{ABSC}(\mathrm{t}+4 \mathrm{n})$ & 0,899996 & 0,00088 & $5-982$ & 7 \\
\hline$C(t+4 n)$ & 0,852933 & $-0,89999$ & $5-982$ & 8 \\
\hline $\operatorname{ABS} C(t+5 n)$ & 0,899996 & 0,00088 & $6-982$ & 9 \\
\hline$C(t+5 n)$ & 0,852933 & $-0,89999$ & $6-982$ & 10 \\
\hline $\operatorname{ABS~C}(\mathrm{t}+6 \mathrm{n})$ & 0,899996 & 0,00088 & $7-982$ & 11 \\
\hline$C(t+6 n)$ & 0,852933 & $-0,89999$ & $7-982$ & 12 \\
\hline $\operatorname{ABSC}(\mathrm{t}+7 \mathrm{n})$ & 0,899996 & 0,00088 & $8-982$ & 13 \\
\hline$C(t+7 n)$ & 0,852933 & $-0,89999$ & $8-982$ & 14 \\
\hline $\mathrm{C}(\mathrm{t})$ & 0,852933 & $-0,89999$ & $1-982$ & 15 \\
\hline $\operatorname{ABSC}(\mathrm{t})$ & 0,899996 & 0,00088 & $1-982$ & 16 \\
\hline
\end{tabular}

Table 2. Output summary of data set.

\begin{tabular}{c|c|c|c|c|c}
\hline & \multicolumn{5}{|c}{ Outputs } \\
\hline Points & $\begin{array}{c}\text { Case } \\
(\boldsymbol{t})\end{array}$ & Case $(\boldsymbol{t}+\boldsymbol{n})$ & $\begin{array}{c}\text { Case } \\
(\boldsymbol{t}+\mathbf{n} \boldsymbol{n})\end{array}$ & $\begin{array}{c}\text { Case } \\
(\boldsymbol{t}+\mathbf{3 n})\end{array}$ & $\begin{array}{c}\text { Case } \\
(\boldsymbol{t}+\boldsymbol{n} \boldsymbol{n})\end{array}$ \\
\hline $1-341$ & 0,06503 & 0,065035 & 0,852933 & 0,852933 & 0,899996 \\
\hline $342-371$ & 0,899996 & 0,899996 & 0,899996 & 0,899996 & 0,779691 \\
\hline $372-467$ & 0,597261 & 0,597261 & 0,597261 & 0,597261 & 0,597261 \\
\hline $468-592$ & 0,587496 & 0,558199 & 0,558199 & 0,558199 & 0,558199 \\
\hline $593-637$ & 0,535550 & 0,559964 & 0,559964 & 0,559964 & 0,559964 \\
\hline $638-733$ & 0,597261 & 0,597261 & 0,597261 & 0,597261 & 0,597261 \\
\hline $734-773$ & 0,54287 & 0,511812 & 0,511812 & 0,504488 & 0,504488 \\
\hline $774-808$ & 0,580171 & 0,608793 & 0,608793 & 0,608793 & 0,608793 \\
\hline $809-876$ & 0,608792 & 0,607023 & 0,607027 & 0,607027 & 0,607027 \\
\hline $877-959$ & 0,425687 & 0,376859 & 0,376859 & 0,376859 & 0,376859 \\
\hline $960-982$ & 0,445218 & 0,445218 & 0,445218 & 0,445218 & 0,445218 \\
\hline
\end{tabular}

sponding those operating conditions in the graphic, the numbers corresponding operating conditions was used as an output. Abstract of the data set acquired was provided in the Table and Table 2. As understood from the Table 1, input data composes of different time intervals of the current and definite values of the current values of these intervals (such as $\mathrm{C}(\mathrm{t}), \mathrm{C}$ $(\mathrm{t}+\mathrm{n})$ etc.) $\mathrm{n}$ value used herein is the sampling time and its value is 0.005 seconds. For the output, conditions of five different time intervals were used. In other words, 16 inputs, neuron number on the 35 hidden layers and 5 outputs were used. (16 35 5). The number of neuron used in the hidden layer was fixed via trial and error method.

Table 3 provides different operating parts of the welding machine according to the data order. Data points shown with red line in the Fig. 5 demonstrate the operating parts in the Table 3.
Table 3. Operation Cases.

\begin{tabular}{c|c}
\hline Data Points & Operation Cases \\
\hline $1-341$ & Initial Case \\
\hline $342-371$ & Transient Case \\
\hline $372-467$ & Optimum Operation Case \\
\hline $468-592$ & Operation Case (downward) \\
\hline $593-637$ & Operation Case (Upward) \\
\hline $638-733$ & Optimum Operation Case \\
\hline $734-773$ & Operation Case (downward) \\
\hline $774-808$ & Operation Case (Upward) \\
\hline $809-876$ & Optimum Operation Case \\
\hline $877-959$ & Operation Case (downward) \\
\hline $960-982$ & Operation Case (Upward) \\
\hline
\end{tabular}

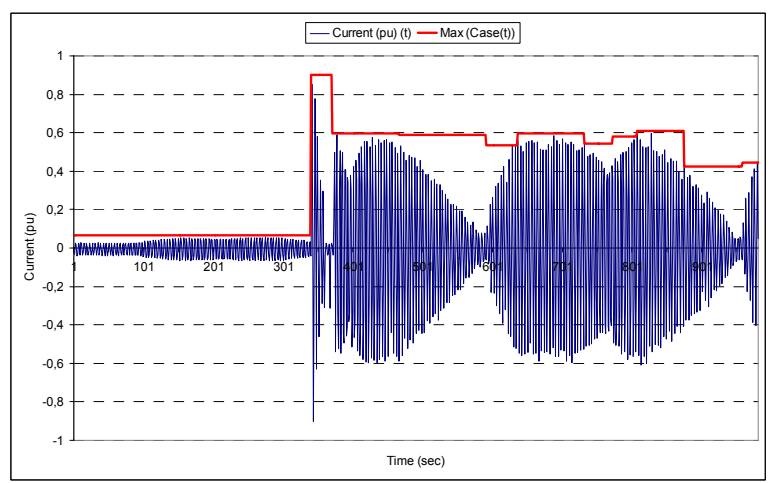

Fig. 5. Overall current variation of the welter.

\section{Normalizing the data}

Normalization of data is a process of scaling the numbers in a data set to improve the accuracy of the subsequent numeric computations and is an important stage for training of the ANN. Normalization also helps in shaping the activation function. For this reason, $[+1,-1]$ normalization function has been used.

\section{Selecting the ANN architecture}

The number of layers and the number of processing elements per layer are important decisions for selecting the ANN architecture. Choosing these parameters to a feed forward back propagation topology is the art of the ANN designer. There is no quantifiable, best answer to the layout of the network for any particular application. There are only general rules picked up over time and followed by most researchers and engineers applying this architecture to their problems. The first rule states that if the complexity in the relationship between the input data and the desired output increases, then the number of the processing elements in the hidden layer should also increase. The second rule says that if the process being modeled is separable into multiple stages, then additional hidden layer (s) may be required. The result of the tests has 


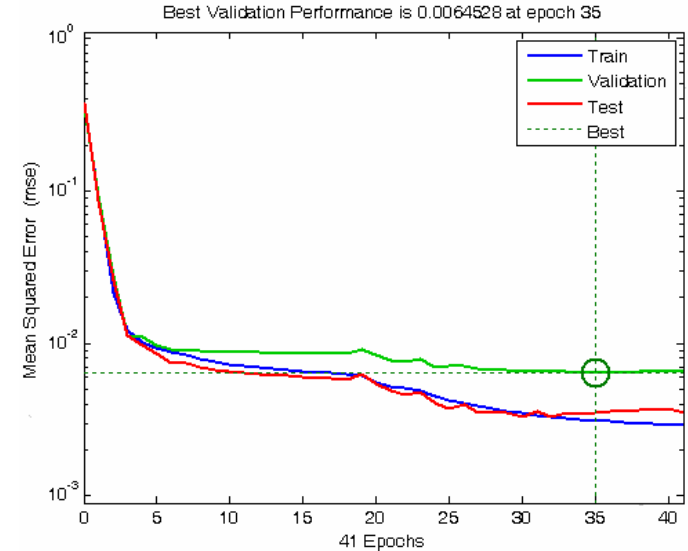

Fig. 6. Variation of the mean square error through the epochs.

showed that the optimal number of neurons in the hidden layer can be chosen as 35 also, the activation function has been chosen as a hyperbolic tangent sigmoid function for all of the layers [18].

\section{Training the network}

In this study ANN was trained with the back propagation (Levenberg - Marquardt) training algorithm. In the training process of this study, the actual output of ANN was compared with the desired output. The training set consists of sixteen input and five output data to the ANN model. The number of data was $982.90 \%$ of this data were used for training. The network adjusted the weighting coefficients that began with random set. The training process has been stopped when the error has become stable. ANN simulator has been trained through the 41 epochs as shown in Fig. 6.

\section{Testing the network}

In the test, an unknown input pattern has been presented to the ANN, and the output has been calculated. Linear regression between the ANN output and target is performed. After ANN learning, test and validation steps founded regression coefficients $(\mathrm{R}=0.981324, \mathrm{R}=0.974999$ and $\mathrm{R}=0.946446)$ shows that target and ANN output values were very related each other. These regression analyses were shown in Fig. 7 for learning step. These coefficient shows that target and ANN output values were very related each other [18]. Also in Table 4, performance of the ANN system is illustrated.

\section{Result and discussion}

For the ANN model formed, 5 outputs were used. There is delay as $\mathrm{n}$ sampling time in these five output interval and its multiples. Such delays were used so as to form test set as input and output of ANN. ANN could be trained in the proportion of $98.13 \%$ according to Table 4.

As a result of the test, a prediction of $97.49 \%$ was carried
Table 4. Results of ANN model.

\begin{tabular}{c|c|c|c|c}
\hline & Samples & MSE & RMSE & R \\
\hline Training & 883 & 0.00313111 & 0.0559563 & 0.981324 \\
\hline Validation & 49 & 0.0064528 & 0.0803293 & 0.946446 \\
\hline Testing & 49 & 0.00353075 & 0.0594201 & 0.974999 \\
\hline
\end{tabular}

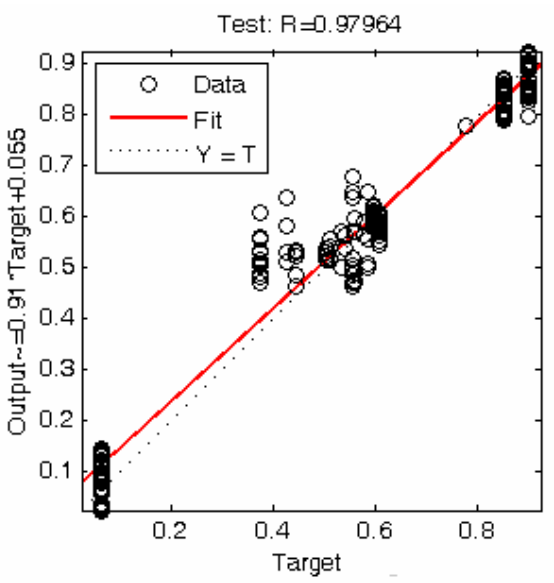

Fig. 7. Regression results of the system.

out according to the R value. As to MSE values, ANN model was successfully trained with value of 0.00313 . In the test performed as a result of this training, MSE values, 0.003530 value was carried out as a successful prediction. A comparison between target desired to be achieved and outputs of ANN model was made using the figure.

It can be understood from the graphics providing all outputs in the Fig. 9(a) and Fig. 9(b) and the graphic demonstrating an output in the Fig. 8(case (t)) that actual value and target value are quite close with each other. Fig. 9 is a three dimensional graphic in which performance of ANN model is acquired through taking into consideration all the outputs collectively. This graphic provides (a) only outputs acquired from ANN model, (b) targets desired to be achieved.

\section{Conclusion}

In this study, a multilayered artificial neutral network model was developed in order to determine the operating parts of an electric Arc welding machine. An empirical application was performed to obtain data set to be used for ANN model. As a result of this test, a five-second current data was obtained. The said current data covers different operating parts of welding machine used for the test during the welding operation. As a result of this study:

The current drawn by a welding machine during welding operation was measured. To this end, a test set was formed.

A time- current analysis was performed by means of the data acquired.

A data set was formed through time series method, based on the time-current graphic.

A multilayered ANN model was developed. This ANN 


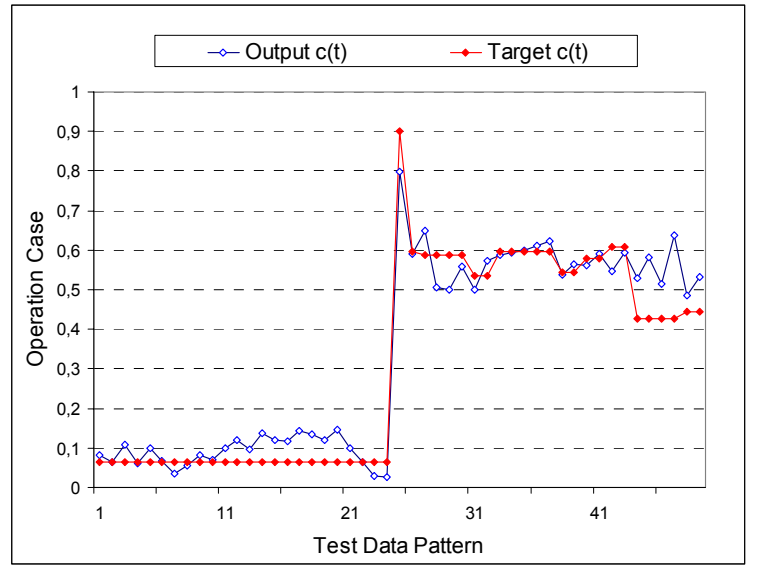

Fig. 8. Variation of the ANN output data together with the target data $(\mathrm{c}(\mathrm{t}))$.

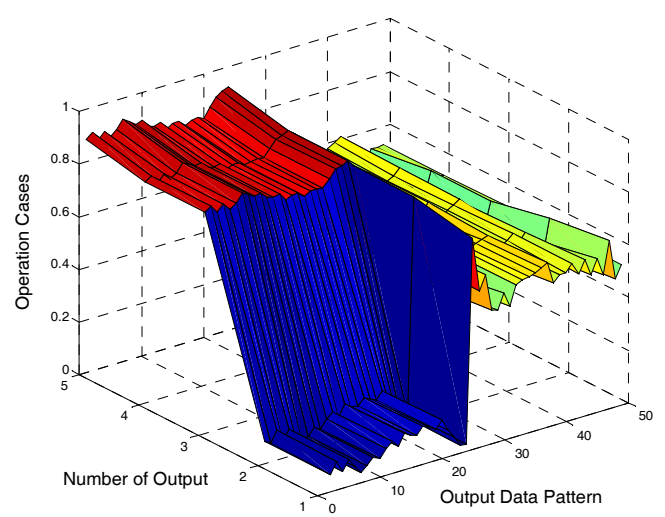

(a)

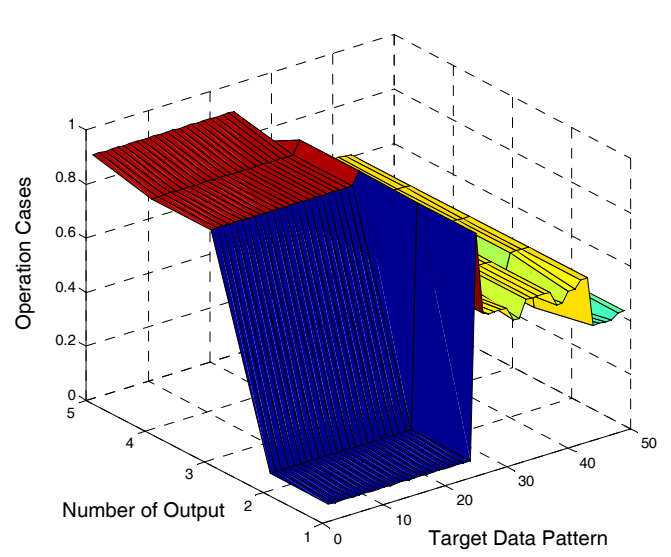

(b)

Fig. 9. Variation of the ANN testing data (a) Output; (b) Target.

model was trained with $90 \%$ of the data set formed via Back propagation learning algorithm. System success was tested with the $10 \%$ of the data set.

As a result of the test, different operating points of the welding machine were predicted with the success of $97.49 \%$.

At the end of the study, it was understood that results of test performed with the electric arc welding machine and ANN outputs were quite consistent with each other.

\section{References}

[1] A. Anders, Tracking down the origin of arc plasma scienceII. early continuous discharges, IEEE Transactions on Plasma Science, 31 (5) (2003) 1060-1069.

[2] Jeff. Nadzam, ed. (1997), Gas Metal Arc Welding Guide lines, Lincoln Electric, http://content.lincolnelectric.com/ pdfs/products/literature/c4200.pdf.

[3] R. Kumar, U. Dilthey, D. K. Dewivedi, S. P. Sharma and P. K. Ghosh, Welding of thin sheet of Al alloy (6082) by using Vario wire DC P-GMAW, The International Journal of Advanced Manufacturing Technology, Springer London, September (2008) 102-117.

[4] S. Goecke and M. Lundin, Tandem Mig/Mag Welding, $A$ Welding Review Published by Esab, 56 (2-3) (2001) 24-28.

[5] MAG Welding with Solid Wire - Methods and Equipment http://www.migatronic.hu/Media/magwelding.pdf, 1-12.

[6] H. B. Cary and C. H. Scott, Modern Welding Technology, Upper Saddle River, New Jersey: Pearson Education. ISBN 0-13-113029-3 (2005) 7-11.

[7] J. E. Harry, Measurement of Elecrical Parameter of AC Arcs, IEEE Transactions on Industry and General Applications, IGA-5 (5) (1969) 594-599.

[8] H. Xinfeng and G. Song, Spectral Analysis of the Plasma in Low-Power Laser/Arc Hybrid Welding of Magnesium Alloy, IEEE Transactions on Plasma Science, 37 (1) (2009) 76-82.

[9] K. Ruth, Welding Basics: An Introduction to Practical \& Ornamental Welding, Creative Publishing International Inc. Minnesota, ISBN: 1-58923-139-2 (2004) 38-44.

[10] J. F. Lancaster, The Physics of Welding, Pergamon Press (1984) 169-172.

[11] L. Jeffus, Welding: Principles and Applications. Albany: Thomson Delmar, ISBN:0-8273-8240-5 (1999) 6-15.

[12] T. M. Hagan, H. B. Demuth and M. Beale, Neural Network Design, PWS Publishing Company, Boston, 1996, 2-44.

[13] G. E. P. Box and G. Jenkins, Time Series Analysis, Forecasting and Control, Holden-Day, San Francisco, CA (1970) 239-256.

[14] Z. Aydogmus, A neural network-based estimation of electric fields along high voltage insulators. Expert Systems with Applications (2009) 36 (4) 8705-8710.

[15] T. M. Hagan, H. B. Demuth and M. Beale, Neural Network Design. PWS Publishing Company, Boston (1996) 2-44.

[16] B. K. Bose, Modern Power Electronics and Ac Drives, Prentice Hall, USA (2002) 625-689.

[17] M. Hamburg, Statistical Analysis for Decision Making, Harcourt, Brace and World Inc., New York (1970) 542-544.

[18] H. S. Nogay, Prediction of Internal Temperature in Stator Winding of Three-Phase Induction Motors with "ANN", European Transactions on Electrical Power. 20:1-9. DOI: 10.1002. 2010 .

[19] http://focus.ti.com/analog/docs/microsite.tsp?sectionId=560 \&tabId=2182\&micrositeId=7, access date 15.06.2010.

[20] W. Koon, Current Sensing for Energy Metering, Technical 
Article, Analog Devices, Inc. (2010) 2-9.

[21] Caddock Electronics, Inc., MP2060 Kool-Pak Clip Mount Power Film Resistor, Data Sheet 28_IL128.1004 (2004) 1.

[22] Texas Instruments, INA199A1-A3EVM, User's Guide SBOU085 (2010) 4-11.

[23] Burr-Brown Corporation, INA146 High-Voltage, Programmable Gain Difference Amplifier, Data Sheet PDS1491A (1999) 1-11.

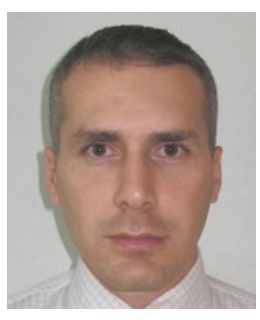

Tahir Çetin Akıncı was born in Pinarbasi, Turkey. He received M.Sc. and Ph.D. degrees from Marmara University of Istanbul, Turkey, in 2002 and 2009 respectively. He is currently assistant professor of electrical and electronics engineering department at Kirklareli University. His research interests are signal processing, control systems, electrical power systems, non-linear dynamical systems, soft computing and condition monitoring techniques.

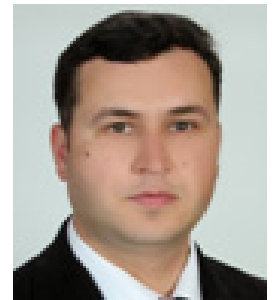

Hidir Selçuk Noğay was born in Isparta, Turkey on 28th june 1975. He received, M.Sc. and Ph.D degrees from Marmara University of Istanbul, Turkey, in 2002 and 2008 respectively. He is currently Director of Kurklareli University Luleburgaz Vocational High School. His research interests include among others renewable energies, electrical machines, electromagnetic fields, vocational technical education and artificial intelligence.

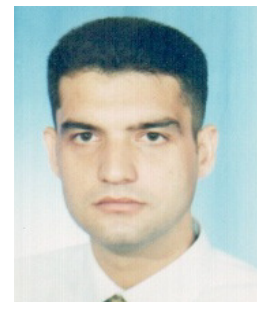

Gökhan Gökmen was born in 1974. He received B.S, M.S and Ph.D. degrees from Marmara University, Istanbul, Turkey. He has been working as an assistant professor at Marmara University. His current interests are measurement method, signal processing techniques, reactive power analysis and compansation syatems, harmonic analysis, vocational education. 